\title{
BMJ Open Rhythm and Movement for Self- Regulation (RAMSR) intervention for preschool self-regulation development in disadvantaged communities: a clustered randomised controlled trial study protocol
}

\author{
Kate E Williams (D), Sally Savage, Rebecca Eager
}

To cite: Williams KE, Savage S, Eager R. Rhythm and Movement for Self-Regulation (RAMSR) intervention for preschool self-regulation development in disadvantaged communities: a clustered randomised controlled trial study protocol. BMJ Open 2020;10:e036392. doi:10.1136/ bmjopen-2019-036392

- Prepublication history for this paper is available online. To view these files, please visit the journal online (http://dx.doi. org/10.1136/bmjopen-2019036392).

Received 14 December 2019

Revised 29 July 2020

Accepted 05 August 2020
Check for updates

(C) Author(s) (or their employer(s)) 2020. Re-use permitted under CC BY-NC. No commercial re-use. See rights and permissions. Published by BMJ.

School of Early Childhood \& Inclusive Education, Faculty of Education, Queensland University of Technology, Brisbane, Queensland, Australia

Correspondence to

Dr Kate E Williams;

k15.williams@qut.edu.au

\section{ABSTRACT}

Introduction Self-regulation (the ability to regulate emotion, attention, cognition and behaviour) is an integral part of early learning competence in the years prior to school. Self-regulation skills are critical to ongoing learning behaviours, achievement and well-being. Emerging neurological evidence suggests coordinated music and movement participation could support selfregulation development for all children. A pilot study in 2016 introduced a coordinated music and movement programme designed to boost self-regulation skills in children in disadvantaged communities, delivered by visiting specialists, with promising findings. The intervention is based on the neuroscience of beat synchronisation, rhythmic entrainment and the cognitive benefits of music therapy and music education - and is called Rhythm and Movement for Self-Regulation (RAMSR) This study builds on the pilot by training regular teachers to deliver RAMSR in their classrooms (rather than visiting specialists). The study aims to establish the effectiveness of RAMSR, which is designed to translate the cognitive benefits that accrue from rhythm participation to address self-regulation for children who do not typically access high-quality music programmes.

Methods and analysis We will recruit 237 children from up to eight kindergartens in low socioeconomic areas. Intervention: teachers will be trained to deliver the RAMSR intervention during group time in kindergartens, daily for 8 weeks. Control: usual practice kindergarten programme. Follow-up: end of intervention using child assessments and teacher report; 12 months postbaseline using school teacher reports following school transition. Primary outcomes: executive function and self-regulation. Secondary outcomes: school readiness; visual-motor integration; teacher-reported behaviour problems, school transition and academic competency; teacher knowledge, confidence, practice and attitudes related to selfregulation, rhythm and movement; fidelity of intervention implementation.

Ethics and dissemination Queensland University of Technology Human Research Ethics Committee, approval 1900000566. Findings dissemination: in-field workshops
Strengths and limitations of this study

This is the first trial to examine the effectiveness of training early childhood teachers to use rhythmic movement to support self-regulation development.

- A wide range of measures including direct assessments and teacher report are used for the outcomes.

- The use of teacher reports for some constructs is a limitation.

- Children will be followed up 1 year later after transitioning to school.

- Maintaining the sample size to the 1-year follow-up will be a challenge.

to service providers, conference presentations, journal and professional publications.

Trial registration number ACTRN12619001342101; Preresults (30 September 2019).

\section{BACKGROUND}

Self-regulation is an umbrella term that includes regulating attention and emotion, and the executive functions including working memory (holding information in mind), shifting (flexible shifting of attention between information or tasks) and inhibition (the ability to control urges and resist distraction). ${ }^{1}{ }^{2}$ These self-regulation skills develop rapidly across early childhood, and a large body of research has now established their predictive association with a range of later life outcomes including educational achievement, ${ }^{3}$ adolescent and adult adaptive behaviours and life success. ${ }^{4}$ Importantly, children with poor early childhood selfregulation skills are at risk of later behaviour problems, ${ }^{6}$ adolescent risk taking ${ }^{4}$ and adult disordered behaviour. ${ }^{5}$ 
It has been estimated that up to $30 \%$ of children aged up to 5 years in Australia may experience self-regulation difficulties including below average attentional and emotional regulation skills that do not improve as expected over time. ${ }^{7}$ A number of life circumstances place children at heightened risk of poorer self-regulation including poverty. ${ }^{8}$ In fact early childhood self-regulatory capacity has been identified as the likely key mechanism through which early life risks including socioeconomic disadvantage lead to poorer academic and well-being outcomes. ${ }^{9} 10$ Specifically, in the context of poverty, household chaos and stress, children's physiology and neurobiology may develop in such a way as to limit their capacity for selfregulation development. ${ }^{11} 12$

Supporting self-regulation development in the years prior to school transition is particularly important. Children with self-regulation difficulties will struggle to pay attention in early learning environments, will remain distressed after negative experiences, will display impulsive behaviours likely to get them in to trouble and will not be able to manage multiple sources of information in complex classrooms. A major problem is that, by school entry, there are already considerable differences in children's self-regulatory abilities, which are thought to be the primary contributors to socioeconomic-related gaps in school readiness. ${ }^{9}$ Children who enter school behind in terms of school readiness typically experience ongoing achievement and well-being gaps that the experience of schooling tends to widen rather than close for most students. ${ }^{13}$

Early intervention to support the development of selfregulation could mediate early socioeconomic risks for children by supporting positive school transition and provide a buffer against poorer academic and mental health outcomes across the school years. ${ }^{29} 14$ A number of curriculum-wide approaches and specific interventions have been implemented and examined for effectiveness.
Three preschool curriculum approaches in the USA studied through randomised control trials (RCTs) have shown mixed effects across studies (eg, Tools of the Mind curriculum), ${ }^{15}$ or positive effects for some self-regulation measures but not others (eg, Head Start Research based developmentally informed (REDI) and Chicago School Readiness Program) ${ }^{16-18}$ Shorter-term targeted interventions implemented either by teachers or visiting specialists within early childhood education and care and studied in RCTs have included the use of picture books with embedded executive function activities ${ }^{19}$ and circle games. ${ }^{20}$ Overall, findings from these specifically designed interventions have been promising but mixed in terms of the effectiveness of the various approaches to impact the various components of self-regulation.

Despite growing evidence about the neurobiological differences underpinning socioeconomic achievement and self-regulation development gradients, ${ }^{19}$ very few interventions have taken a neurobiological approach to addressing early childhood self-regulation. In 2016, we designed and piloted an intervention (called Rhythm and Movement for Self-Regulation (RAMSR)) that aimed to leverage the known neurological benefits of formal music training and clinical applications of music therapy, through delivery in mainstream early childhood education settings by teachers. Distinct synergies across a range of research areas, that we have reviewed elsewhere, ${ }^{21}$ suggested that a specifically designed rhythm and movement intervention that used rhythmic auditory cueing and focused on beat synchronisation, coordinated rhythmic motor and executive function activities, would have the potential to improve early childhood self-regulatory skills with implications for longer term behavioural and school transition outcomes (see figure 1).

The RAMSR pilot was a quasi-experimental clustered trial in three kindergarten centres in disadvantaged communities, with two classrooms in each centre. ${ }^{22}$ One

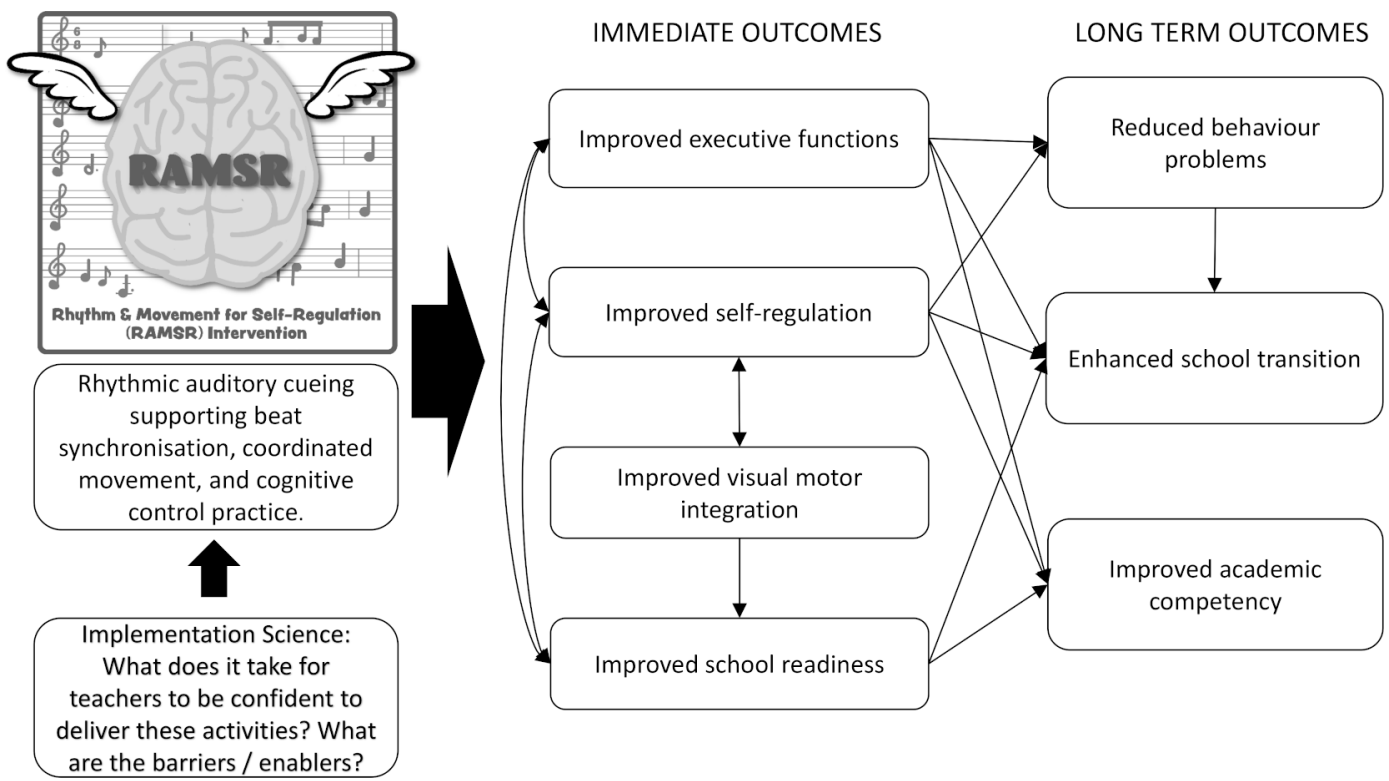

Figure 1 Intervention logic model for Rhythm and Movement for Self-Regulation (RAMSR). 
classroom in each centre was non-randomly assigned to intervention, and one to control, based on classroom schedules aligned with availability of visiting RAMSR specialists delivering the programme. Results showed positive intervention effects for teacher-reported emotional regulation growth (moderate effect size of 0.35 ) and the directly assessed executive function of shifting for boys (large effect size of 0.60). ${ }^{22}$ Fidelity ratings by visiting specialists delivering RAMSR showed that activities were implemented in accordance with the plan for $77 \%-98 \%$ of instances, depending on the activity and child enjoyment was rated as high for $77 \%$ of all sessions conducted.

This study will build on this previous trial by investigating the effectiveness of the same intervention as delivered by teachers, rather than visiting specialists. For activities with lower levels of fidelity across the pilot, these were adjusted and redesigned in preparation for this RCT, based on the qualitative notes provided by implementation leaders during the pilot. New evidence that will be produced by this RCT is important to establish if the intervention is to be scaled up and increase its reach with the more sustainable approach of supporting the existing teachers to deliver the programme. While training teachers to deliver interventions with fidelity has challenges, ${ }^{23}$ this study will use best evidence in teacher professional development ${ }^{24}$ to ensure fidelity and quality of intervention implementation. Specifically, the training takes an experiential model, followed up with supports including coaching and a community of practice. An implementation science approach will be used to understand the enablers and barriers to teachers implementing the intervention within their usual programming, and what is needed to support their confidence and skill to do so. The research questions for the trial are:

1. How effective is a short-term (16-20 sessions of 20 minutes each over 8 weeks) rhythm and movement intervention delivered by teachers in improving the self-regulation skills of preschool children (3-4years old) and are any improvements sustained following the transition to school ( $4-5$ years old)?

2. How well does the training and resources provided support teachers to implement the programme with fidelity and increase their confidence to practice rhythm and movement activities with young children?

We hypothesise that compared with the control children at 10 weeks and 12 months post baseline, the 8-week RAMSR intervention delivered by teachers in the year prior to school will:

A. Improve children's self-regulation and executive function skills including components of emotional regulation, attentional regulation, behavioural regulation, classroom regulation and executive functions of shifting, working memory and inhibition.

B. Be related to a range of secondary outcomes for children including improved school readiness and visual-motor integration, fewer behaviour problems and enhanced school transition.
We also expect that compared with teachers not trained in the intervention, teachers trained in RAMSR will:

C. Show an increase in teacher knowledge, confidence, practice and attitudes related to self-regulation and rhythm and movement compared with their baseline measures.

Finally, we hypothesise that:

D. The proposed training and coaching support for teachers will result in high rates of intervention fidelity defined as ratings of 'implemented according to plan or with planned extensions' for $80 \%$ or more of the implementation instances for each activity across the programme.

\section{METHODS \\ Design}

The project will use a balanced-clustered RCT design to follow one cohort of children from kindergarten programme entry (2020) and into the first year of school (Preparatory; 2021).

\section{Setting}

In Queensland, Australia, the year prior to formal school commencement is known as kindergarten. Participation rates in this year are over $95 \%$ nationally ${ }^{25}$ and children attend either a long day care centre, with the potential to attend up to 12 hours per day 5 days per week (as enrolled by their family), or a stand-alone kindergarten programme that typically offers either a 5-day fortnight programme (6hours per day) or a 2-day per week programme with longer days ( 7.5 hours per day). In stand-alone kindergartens, the same cohort of children is enrolled in each programme, as opposed to long day care where different groups and combinations of children attend on any given day of the week. The national curriculum for the years prior to school, the Early Years Learning Framework, ${ }^{26}$ guides the play-based approach in both types of programmes. All kindergarten programmes employ a bachelor-qualified teacher and a teaching assistant as well as other inclusion support educators as needed. The adult to child ratio is $1: 11$, with stand-alone kindergartens generally enrolling up to 22 children per group. Children are typically $3.5-4.5$ years at commencement of the kindergarten year and enrol in kindergarten for 12 months.

We will engage with one major early childhood education and care service provider (Childcare \& Kindergarten Association; C\&K) to support recruitment of kindergartens. C\&K, established in 1907, is a Queensland based, large, not-for-profit organisation with 350 kindergartens and childcare centres. C\&K manages two types of services-branches and affiliates. Branches are where $\mathrm{C} \& \mathrm{~K}$ is the licensee of the service and responsible for the management of staff, workplace issues, insurances, information technology, legislative compliance, buildings and maintenance and funding. ${ }^{27}$ Affiliate centres have entered into a service agreement with $\mathrm{C} \& \mathrm{~K}$, where C\&K 
distributes funding, provides support and audits compliance obligations on behalf of the Queensland Government. ${ }^{28} \mathrm{C \& K}$ is providing no financial or other resources to the project and is not being paid for their collaboration in centre recruitment. A formal research approval process with C\&K has been completed.

Centres to be recruited will meet the following criteria: affiliate or branch member with C\&K; located within $70 \mathrm{~km}$ from Brisbane, Australia; be in a low socioeconomic area as indicated by a score in the bottom three deciles of a national index based on census data that position areas in Australia according to relative socioeconomic advantage and disadvantage ${ }^{29}$; be in a community area where 2018 Australian Early Development Census data indicate a higher than national average level of child vulnerability in both domains of social competence and emotional maturity ${ }^{30}$; run a kindergarten programme with the same cohort of children attending at least 2 consecutive days per week (ie, stand-alone kindergarten rather than long day care); have a teacher in the kindergarten programme who is not planning to retire, resign, transfer or take long service leave during the trial period (last quarter 20192020); have a typical start-of-year enrolment of at least $80 \%$ of capacity in each group (17 children in a group with capacity to take 22).

\section{Kindergarten teacher recruitment}

Directors at all eligible centres will be emailed trial information by C\&K and invited to complete an expression of interest form, which will collect information further checking on eligibility items (including anticipated teacher employment stability). From those centres who complete an expression of interest, an independent researcher not involved in the study will randomly select the study centres, and teachers within the study centres, using stratification of child enrolment capacity, provided by teachers on the expression of interest form. Some teachers will teach up to 22 children, if they teach in one programme, and up to 44 children, if they teach in two programmes, across the week. If multiple teachers from one centre return an expression of interest, only one teacher from each centre will be randomly selected to reduce the introduction of another level in analyses. The independent researcher will select an even number of teachers with 22 children and teachers with 44 children to ensure a balanced design across two conditions is possible. As well as sociodemographic and context measures (table 1), all teachers will complete a baseline survey collecting information on outcome measures of frequency of music, rhythm and movement practices; confidence in using rhythm and movement; level of knowledge on self-regulation and music and movement for brain development; beliefs about the role of active music engagement for development; training and experience in education and specifically in self-regulation and rhythm and movement (table 2).

\section{Randomisation}

Randomisation will occur at the centre level (which is also the teacher level) following recruitment of centres and the teachers within the centre and collection of baseline teacher data. An independent researcher will use a random number generator again stratifying by teacherprovided child enrolment numbers to ensure a balanced number of children in both intervention and control groups. This is because teachers will either teach 22 children or 44 children across a given week depending on their centre or work arrangements as described above. Stratification will mean an equal number of smaller units and larger units will be assigned to each condition to achieve a balanced design. Intervention teachers will then train in programme delivery, practicing with their 2019 preschool cohort. In 2020, a new cohort of preschool children will be enrolled in the centres and invited to participate in the research, meaning randomisation must occur prior to child recruitment and baseline measures.

\section{Intervention training}

Teachers in the intervention centres will be provided with intervention materials and professional development in the form of brief self-paced online orientation covering the rationale for the programme; full-day workshop covering self-regulation in early childhood, the theory and rationale for use of rhythm and movement and exposure to all of the intervention materials, microteaching demonstrations of the intervention (teachers act as children so they can experience the activities themselves); a demonstration led by the research team in their centre; independent practice of sessions with their 2019 class; observation and coaching by the research team; and engagement with the online professional learning community for tip sharing and trouble shooting. Prior to the implementation period, an online refresher course will be completed. In 2021, following the completion of data collection, all control teachers will gain access to RAMSR materials and training.

\section{The intervention}

RAMSR is designed to be delivered by early childhood practitioners in a group setting with up to 25 children. Teachers will deliver this as part of their usual group floor or circle time within the kindergarten daily routine. Typically, this is held in the morning as a warm up to the day. The RAMSR programme consists of a series of four session plans, with each plan to be repeated daily for 2 weeks. In these settings, because of enrolment patterns, this will mean each child receives the programme four to five times per fortnight, yielding a total of 16-20 sessions available to each child over the 8-week intervention period.

All activities within the session plans are designed to practice key skills of attentional, emotional and behavioural regulation, inhibition, shifting and working memory through embedding these skills in coordinated movement activities enhanced by rhythmic auditory cueing. Common activity elements across the sessions included start/stop (inhibition), reversal of instruction (shifting, for example, move in the silence and freeze in the music), working memory games and beat 
Table 1 Sociodemographic, context, education training and intervention fidelity measures to be used in the study

\begin{tabular}{|c|c|c|c|c|}
\hline Construct & Measure & Baseline & $\begin{array}{l}8 \text { weeks } \\
\text { follow-up }\end{array}$ & $\begin{array}{l}12 \text { months } \\
\text { follow-up }\end{array}$ \\
\hline \multirow[t]{2}{*}{$\begin{array}{l}\text { Sociodemographic } \\
\text { characteristics }\end{array}$} & $\begin{array}{l}\text { Educator/teacher: level of education, years of } \\
\text { experience, employment status, age, gender }\end{array}$ & 0 & & ○ \\
\hline & $\begin{array}{l}\text { Child/parent: age, gender, language and } \\
\text { indigenous background, income bracket, } \\
\text { household composition, parental education, } \\
\text { child disability or delay }\end{array}$ & 䊑 & & \\
\hline
\end{tabular}

Preschool educator context

$\begin{aligned} \text { Music training background } & \text { Study-designed questions on type and years } \\ & \text { of formal music training as well as music } \\ & \text { training as part of preservice and in-service } \\ & \text { professional development (six items) }\end{aligned}$

Self-regulation training Study-designed questions on type of background preservice and in-service professional development in area of self-regulation (four items)

Child context measures

Frequency of music-related Single item about frequency of home adulthome learning child music activities used in prior longitudinal studies $^{35}$

\begin{tabular}{|c|c|}
\hline $\begin{array}{l}\text { Attendance at } \\
\text { extracurricular music and/ } \\
\text { or dance }\end{array}$ & $\begin{array}{l}\text { Single item asking about music/dance } \\
\text { extracurricular in the last } 6 \text { months }\end{array}$ \\
\hline Sleep problems & Five items used in prior longitudinal studies ${ }^{36}$ \\
\hline $\begin{array}{l}\text { Physical activity preference } \\
\text { and levels }\end{array}$ & $\begin{array}{l}\text { Four items from the Longitudinal Study of } \\
\text { Australian Children }{ }^{37} \text {-two from each of parents } \\
\text { and school teachers } \\
\text { Parents: one item on a 5-point scale about } \\
\text { child enjoyment of physical activity from } \\
\text { very much dislikes to very much likes; one } \\
\text { item about child's use of free time with three } \\
\text { categories related to whether chooses active, } \\
\text { inactive or both pastimes. } \\
\text { School teacher: two items asking on a 5-point } \\
\text { scale how active child is in organised activities } \\
\text { and in play (from a lot less active than most to } \\
\text { a lot more active than most) }\end{array}$ \\
\hline
\end{tabular}

Intervention feasibility, acceptability and fidelity measures and teacher training/delivery experiences (intervention group only)

$\begin{array}{ll}\text { Implementation and } & \text { Sessional checklist against activity plan. Each Throughout programme } \\ \text { adjustment record } & \text { of the six activities undertaken in each section delivery } \\ & \text { will require a rating by teachers of either 'as per } \\ \text { plan with or without planned extensions'; 'as } \\ \text { per plan with adjustments'; 'not implemented' }\end{array}$

Teacher engagement Sessional checklist on teacher enjoyment and confidence (low, medium, high)

Throughout programme delivery

\begin{tabular}{lll} 
Child engagement & $\begin{array}{l}\text { Session checklist on child attention, enjoyment } \\
\text { and participation (low, medium, high) }\end{array}$ & $\begin{array}{l}\text { Throughout programme } \\
\text { delivery }\end{array}$ \\
$\begin{array}{ll}\text { Teacher experience training } \\
\text { in and delivering the }\end{array}$ & $\begin{array}{l}\text { Individual narrative interview including } \\
\text { intervention }\end{array}$ & $\begin{array}{l}\text { barriers and facilitators to approach and } \\
\text { implementation }\end{array}$ \\
& $\begin{array}{l}\text { Coach observation and coaching discussion } \\
\text { notes }\end{array}$ & $\begin{array}{l}\text { Throughout programme } \\
\text { training and delivery }\end{array}$ \\
\hline
\end{tabular}

$\bullet=$ direct assessment; $\boldsymbol{\theta}=$ =preschool educator report; $*=$ =parent report; $\mathbf{0}=$ =school teacher report. 
Table 2 Kindergarten educator outcome measures to be used in the study

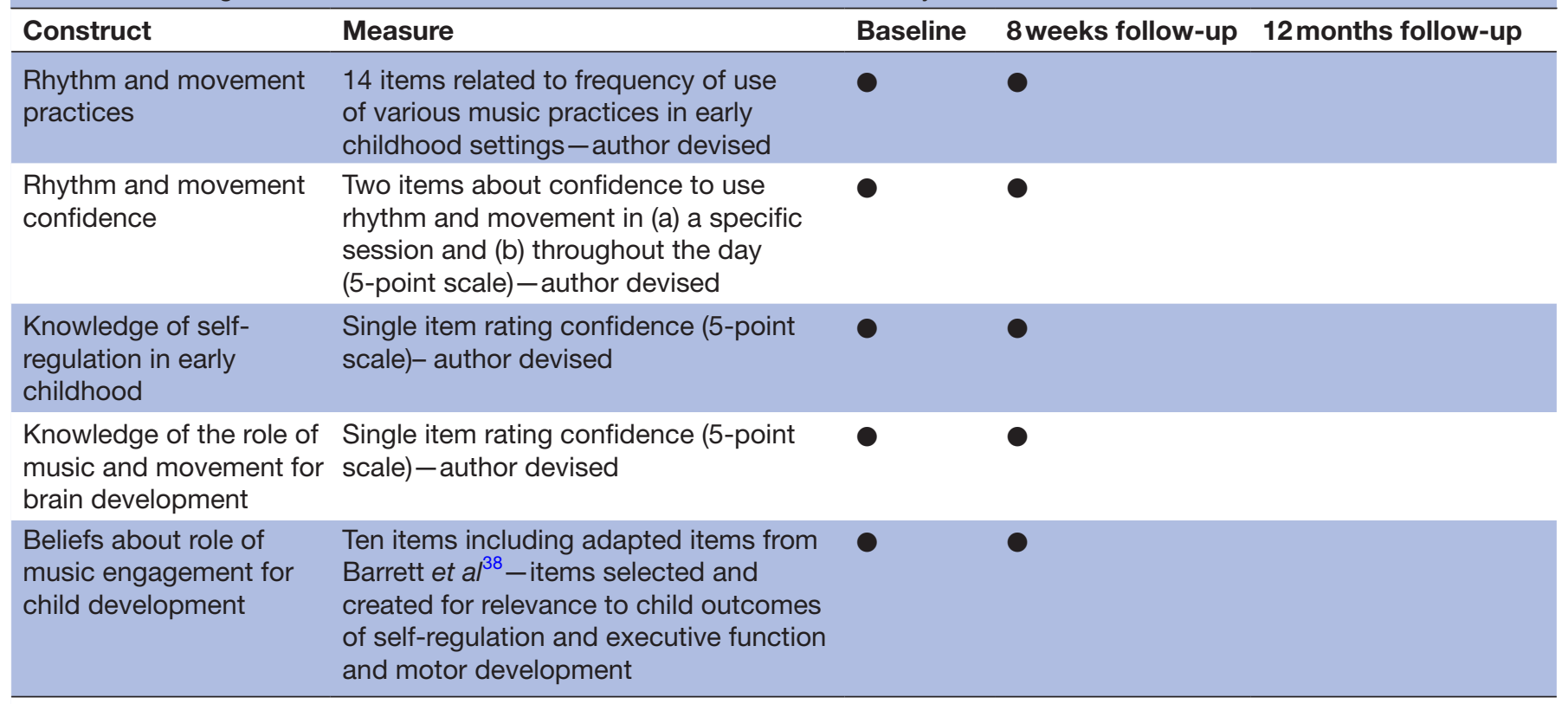

=preschool educator report

synchronisation to changing tempos. Original backing tracks provided rhythmic auditory cueing and leverage rhythmic entrainment principles to stimulate more coordinated movement.

Within each session plan, there are a series of activities with the above key elements represented in each (1) warm-up involving body percussion, (2) becoming familiar involving an adaptation of a familiar early childhood song, (3) moving to the beat involving large gross motor movements, (4) playing to the beat involving simple rhythm sticks or castanets, (5) dancing to the beat involving slightly more complex gross motor movement patterns to activity three of the session and often involving visual-motor skills and coordination such as mirroring the shape of rhythm sticks on the floor with bodies and (6) calming, which includes a series of movements and then stillness accompanied by relaxation music to support physiological entrainment to a calmer state, targeting embodied emotional regulation. Teachers are provided with each session plan, a series of videos demonstrating the plans, all audio tracks required to run the activities and visual cue cards needed for some activities. Rhythm sticks and animal castanets required to implement the sessions are provided. Further and regularly updated information on RAMSR, including training approaches and opportunities, can be found at https://ramsrblog. wordpress.com/.

\section{Child recruitment and baseline measures}

Teachers will invite all children enrolled and commencing the 2020 kindergarten year between 28 January 2020 and 21 February 2020 (first 4 weeks of the kindergarten year) to participate in the research through gaining parental consent. Validated baseline self-regulation measures (parent and teacher report and direct assessments with children) will be taken with all children prior to the intervention/control period (baseline; see table 3). Assessors will visit kindergartens and withdraw children to undertake assessments and will be blind to group assignment. Assessors will visit centres in pairs, with each child working with each assessor for a 15-minute session, splitting the tasks into two sessions. Kindergarten teachers will provide checklist measures of children and will not be blind to group assignment.

\section{Intervention delivery}

In term 1, teachers will undertake a brief refresher training seminar ( 2 hours) prior to commencing delivery of the programme 2-3 times weekly for 8 weeks. Teachers will access ongoing support through regular phone contact and access to the closed online professional learning community. Once every 2 weeks, a research team member will visit to observe the teacher delivering the session, to take fidelity measures and to provide coaching. Coaching will follow protocol by Hemmeter et $a l^{31}$ Each postsession reflection will involve: open, summarise observation and encourage reflection; supportive performance feedback; corrective performance feedback; targeted support; support for planned actions; end meeting. A summary of each verbal coaching session will be provided via email to teachers and this will become a source of research data to address our second research question. Teachers (and coaches when observing) will provide fidelity checklist information following the completion of each session, which will collect data on: adherence to the session plan; level of child engagement in sessional activities as a measure of participant responsiveness; attendance data of children to determine dosage. The research team lead 


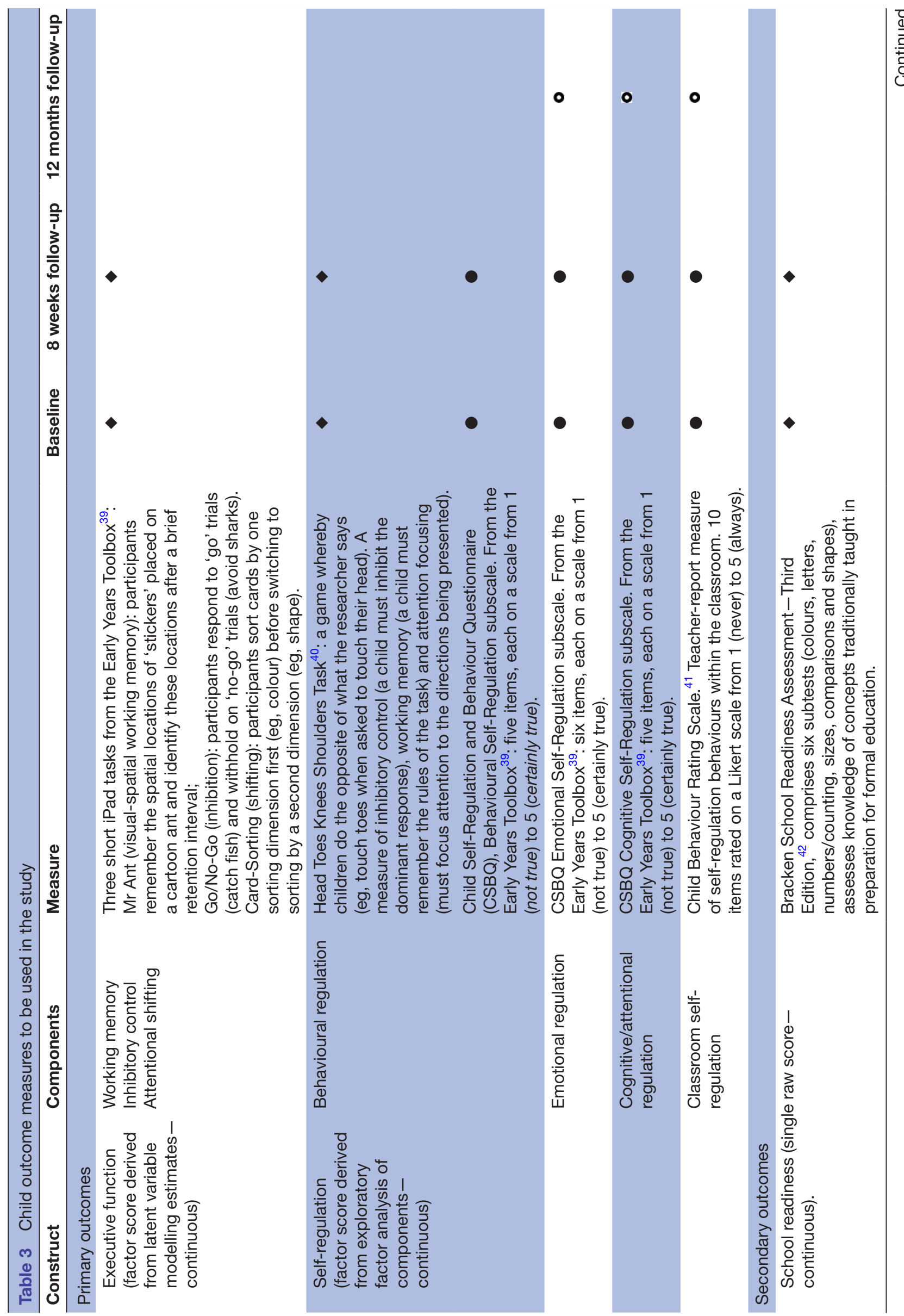




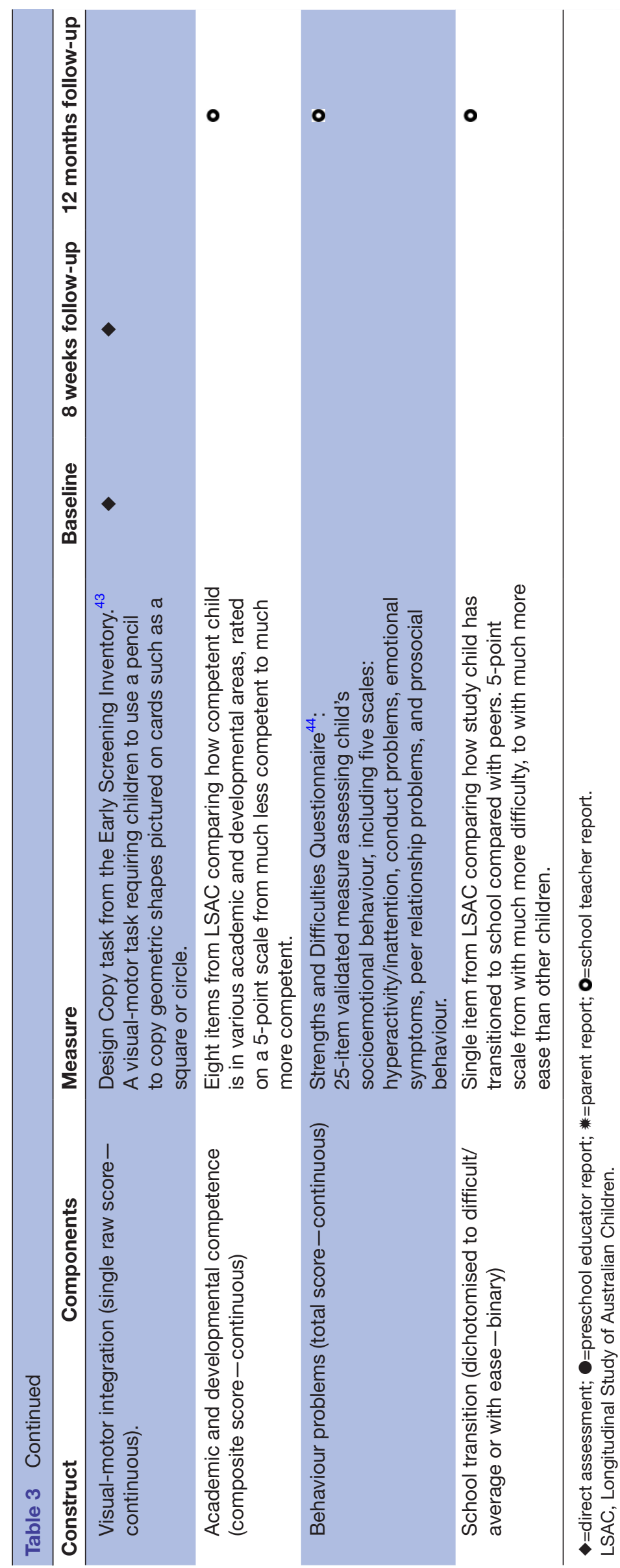


will also make additional visits to all pairs of teachers and coaches to collect additional independent fidelity checklist information. Programme differentiation across the control and intervention groups will be ascertained through examination of baseline and follow-up data collected on teacher surveys regarding the frequency of use of rhythmic movement sessions in kindergartens.

\section{Follow-up}

Follow-up data collection will occur at the end of the 8-week intervention period, and 12 months after baseline, after children have transitioned to school (table 2). All participating families will be telephoned early in 2021, when most children will be enrolled in Prep (first year of full-time schooling in Queensland). Parents will be asked to provide contact details for their child's school and current teacher and to confirm consent for the research team to contact the school. Prep teachers will then be asked to complete the self-regulation measure for each child (paper form delivered to the school), providing follow-up data for children following school transition. School teachers will be blind to group assignment. Teacher report has been selected as the only data collection approach at the 12-month follow-up period, rather than a repeat of direct child assessments undertaken at baseline and 8-week follow-up. This is because it is anticipated that study children will attend up to 50 different schools spread across a wide geographic region and it is not feasible within financial constraints of the study to undertake direct child assessments. However, as depicted in table 3 , four of the teacher-report measures are repeated across all three time points, which will allow for some consistency in the analyses.

\section{Measures}

The measures used throughout the study are summarised in tables 1-3. Measures have been selected with reference to the known psychometric properties and feasibility of measures in low socioeconomic kindergarten settings, many of which were used in the pilot study for the current trial. ${ }^{22}$ The project lead will provide training for face-toface measures to the data collection team. The training will involve familiarisation with the measure's components and how they are administered. Mock assessments will be conducted to further familiarise the staff in the presence of the trainer. The lead researcher will also observe each assessor once in the first week of data collection, and ongoing troubleshooting meetings will be held to ensure fidelity.

All electronic and paper data will be collected against a unique digital identifier for each participant. Data will be directly uploaded to a database for the three iPad tasks. The Design Copy task will be double scored (blind) by the lead author and a single research assistant, with inter-rater reliability calculated. For the two other child assessment measures, data will be collected through data collection paper forms. Data entry will be managed by a single data entry research assistant with a $10 \%$ random selection of forms for each measure double checked by the lead author. Confidentiality is ensured through storage of all data against numeric identifier codes only. The identification key will be stored separately and only accessible by the lead researcher and senior research assistant.

\section{Sample size}

Children: pilot study estimates have been used for power and sample size calculations. Assuming a simple sampling design with no clustering, calculation in $\mathrm{G}^{*}$ Power indicates a required sample size of 76 to detect small intervention effects (0.14) with a power of 0.8 using the planned analytic approach (path analysis equivalent to multiple regression). The balanced cluster design of the RCT produces a design effect of up to 1.9 (using average intraclass correlation coefficients for same measures used in the pilot of 0.03 and average cluster size of 33), increasing the effective sample size to 144 . Using the 2016 pilot response rates at similar centres as a guide, a sufficient final sample of at least 144 at the 12 month follow-up time point will be achieved by recruiting a minimum of 200 children at baseline (allowing for a 15\% attrition rate at each of the two follow-up time points).

Teachers: with only up to eight teachers involved in the study, split across intervention and control, the study is underpowered to address hypothesis $\mathrm{C}$ using significance testing. Instead, we will use a match case-series approach and descriptive statistics to take an exploratory approach to understanding any baseline similarities and differences in the teacher constructs among intervention teachers and matched control teachers and change scores in constructs across baseline to follow-up.

\section{Statistical analyses}

The efficacy of the intervention will be evaluated within a structural equation modelling framework (using MPlus) with an intention-to-treat approach, which will allow for: precision in the testing of intervention effects on the outcome measures across the intervention and control groups while controlling for baseline scores and covariates; handling of missing data using full information maximum likelihood and accounting for the clustered design. ${ }^{32}$ To reduce the number of analyses undertaken, multiple measures for each construct will be reduced to single scores using appropriate approaches for each as shown in table 3 . For primary outcomes (executive function and self-regulation) and the secondary outcomes measured equivalently at baseline and 8 weeks later (school readiness and visual-motor integration), four separate path analyses (equivalent to multiple regression) will be used with effect sizes computed using formulas for independent groups pre-post designs. ${ }^{33}$ To examine 12-month follow-up data, latent curve modelling for pre-post-post designs will also be used to examine intervention effects across baseline to postintervention to follow-up ${ }^{34}$ for self-regulation factor scores that are equivalently measured at three time points. For the secondary outcomes measured only at 12-month 
follow-up (academic competence, behaviour problems and school transition), path models examining the effect of treatment condition on these outcomes, controlling for baseline measures and mediated by 8 -week follow-up measures will be developed. Clustering will be accounted for at the centre level. Covariates will be selected from child gender and age, level of family income, parental education, non-English speaking status and aboriginal status. Selection will be based on consistency and significance of bivariate correlations among these covariates and outcomes measures.

The first phase of analysis will be undertaken once baseline and postintervention data collection is complete. A second phase of analysis will be completed once the 12-month follow-up data collection is complete.

\section{Participant and public involvement}

Participants and the public were not involved in the design or reporting of this research protocol and will not be involved in the design, reporting and dissemination of the trial.

\section{DISCUSSION}

A positive transition to school provides children with an important advantage in terms of student-teacher and peer relationships and future academic success. A key feature of school readiness is the ability to regulate one's own emotions, cognitions and behaviour. Self-regulatory growth in early childhood is a key indicator of children's capacity to learn and thrive in environments such as school and is linked to better life outcomes more broadly, including educational achievement, employment and well-being. Importantly, children from disadvantaged backgrounds may experience early home environments that are less conducive to self-regulatory growth, which confers vulnerability to poor school transition and subsequently poorer learning and well-being trajectories. With research showing that almost a third of Australian children start school with poor self-regulation skills which can lead to poor life outcomes, it is imperative that this issue is addressed.

RAMSR aims to combine the researched benefits of music education and music therapy, to build the underlying neural pathways that support self-regulatory skills in young children. The initial outcomes of this programme will present as teacher-reported improvements in selfregulation in the form of emotional, attentional and general classroom regulation and improved assessed behavioural regulation and executive functioning. Important secondary benefits will include improved school readiness and transition, learning competency and academic achievement and enhanced kindergarten teacher knowledge and self-efficacy related to rhythm and movement and self-regulation.

Other important attributes of the RAMSR programme are its low cost and accessibility, making it viable for all preschools to use in their everyday programmes. Teachers, even those who consider themselves to be 'non-musical', can implement the programme through a structured and accessible training procedure. Once the programme is trialled, if proven to be effective, it is anticipated that RAMSR will be able to be scaled up to a range of early childhood settings and translated internationally. Further, the study will contribute important information about the particular barriers and facilitators that early educators face in the implementation of specific intervention programmes as they aim to scale up. Increased understanding of implementation factors will be of benefit to educational intervention designers that seek to embed enablers and address potential barriers as part of design and implementation processes.

\section{Ethics and dissemination}

Ethics approval has been granted by the Queensland University of Technology Human Research Ethics Committee, approval 1900000566. During the trial, only the named investigators, senior research assistant, and research student on the project will have access to the dataset. An authorship plan has been negotiated with all project team members. Findings from this project will be disseminated through in-field workshops to key early childhood service providers; national and international conference presentations; academic journal publications and professional early childhood publications. A research brief summarising the findings will be disseminated to participating research sites, along with the research partner, C\&K Association and peak bodies Early Childhood Australia and The Australian Music Therapy Association. Any changes to this protocol will be updated through the trial registration and communicated with all investigators and participants.

Collaborators Childcare \& Kindergarten Association (C\&K): Cathy Nielson; Donna Berthelsen.

Contributors KEW led all aspects of RCT design and the writing of the protocol paper, SS and RE made substantial contributions to intervention design and materials.

Funding This study was funded by Australian Research Council (ARC) Competitive Grant Project ID DE190101096 - secured.

Competing interests KEW reports a grant from Australian Research Council. SS reports work as a paid casual relief teacher for the collaborating agency, Childcare \& Kindergarten Association, for approximately 30 days per year at various sites. This agency has no financial interest in the research project, they are providing access to research sites only.

Patient and public involvement Patients and/or the public were not involved in the design, or conduct, or reporting, or dissemination plans of this research.

Patient consent for publication Not required.

Provenance and peer review Not commissioned; externally peer reviewed.

Open access This is an open access article distributed in accordance with the Creative Commons Attribution Non Commercial (CC BY-NC 4.0) license, which permits others to distribute, remix, adapt, build upon this work noncommercially, and license their derivative works on different terms, provided the original work is properly cited, appropriate credit is given, any changes made indicated, and the use is non-commercial. See: http://creativecommons.org/ licenses/by-nc/4.0/.

ORCID iD

Kate E Williams http://orcid.org/0000-0001-8983-5503 


\section{REFERENCES}

1 Blair C, Raver CC. Individual development and evolution: experiential canalization of self-regulation. Dev Psychol 2012;48:647-57.

2 Diamond A. Why improving and assessing executive functions early in life is critical. In: Griffin JA, McCardle P, Freund LS, et al, eds. Executive function in preschool-age children: integrating measurement, neurodevelopment, and translational research. Washington DC, US: American Psychological Association, 2016: 11-43.

3 McClelland MM, Acock AC, Piccinin A, et al. Relations between preschool attention span-persistence and age 25 educational outcomes. Early Child Res Q 2013;28:314-24.

4 Howard SJ, Williams KE. Early self-regulation, early self-regulatory change, and their longitudinal relations to adolescents' academic, health, and mental well-being outcomes. J Dev Behav Pediatr 2018;39:489-96.

5 Slutske WS, Moffitt TE, Poulton R, et al. Undercontrolled temperament at age 3 predicts disordered gambling at age 32 : a longitudinal study of a complete birth cohort. Psychol Sci 2012;23:510-6.

6 Sawyer ACP, Miller-Lewis LR, Searle AK, et al. Is greater improvement in early self-regulation associated with fewer behavioral problems later in childhood? Dev Psychol 2015;51:1740-55.

7 Williams KE, Nicholson JM, Walker S, et al. Early childhood profiles of sleep problems and self-regulation predict later school adjustment. Br J Educ Psychol 2016;86:331-50.

$8 \mathrm{Li} \mathrm{M}$, Riis JL, Ghazarian SR, et al. Income, family context, and self-regulation in 5-year-old children. J Dev Behav Pediatr 2017;38:99-108.

9 Blair C, Raver CC. School readiness and self-regulation: a developmental psychobiological approach. Annu Rev Psychol 2015;66:711-31.

10 Dilworth-Bart JE. Does executive function mediate SES and home quality associations with academic readiness? Early Child Res $Q$ 2012;27:416-25.

11 Farah MJ. The neuroscience of socioeconomic status: correlates, causes, and consequences. Neuron 2017;96:56-71.

12 Blair C, Raver CC, Granger D, et al. Allostasis and allostatic load in the context of poverty in early childhood. Dev Psychopathol 2011;23:845-57.

13 Rorris A. Australian schooling - the price of failure and reward for success. Australia Education Union, 2016.

14 Crespo LM, Trentacosta CJ, Udo-Inyang I, et al. Self-Regulation mitigates the association between household chaos and children's behavior problems. J Appl Dev Psychol 2019;60:56-64.

15 Solomon T, Plamondon A, O'Hara A, et al. A Cluster RandomizedControlled Trial of the Impact of the Tools of the Mind Curriculum on Self-Regulation in Canadian Preschoolers. Front Psychol 2017;8:2366.

16 Jacob R, Parkinson J. The potential for school-based interventions that target executive function to improve academic achievement. Rev Educ Res 2015;85:512-52.

17 Bierman KL, Nix RL, Heinrichs BS, et al. Effects of head start REDI on children's outcomes 1 year later in different kindergarten contexts. Child Dev 2014;85:140-59.

18 Jones SM, Bub KL, Raver CC. Unpacking the black box of the CSRP intervention: the mediating roles of Teacher-child relationship quality and self-regulation. Early Educ Dev 2013;24:1043-64.

19 Howard SJ, Powell T, Vasseleu E, et al. Enhancing Preschoolers' Executive Functions Through Embedding Cognitive Activities in Shared Book Reading. Educ Psychol Rev 2017;29:153-74.

20 Duncan RJ, Schmitt SA, Burke M, et al. Combining a kindergarten readiness summer program with a self-regulation intervention improves school readiness. Early Child Res Q 2018;42:291-300.

21 Williams KE. Moving to the beat: using music, rhythm, and movement to enhance self-regulation in early childhood classrooms. Int J Early Childhood 2018;50:85-100.

22 Williams KE, Berthelsen D. Implementation of a rhythm and movement intervention to support self-regulation skills of preschoolaged children in disadvantaged communities. Psychol Music 2019:47:800-20.
23 Larson M, Cook CR, Fiat A, et al. Stressed teachers don't make good implementers: Examining the interplay between stress reduction and intervention fidelity. School Ment Health 2018;10:61-76.

24 Snyder P, Hemmeter ML, Meeker KA, et al. Characterizing key features of the early childhood professional development literature. Infants Young Children 2012;25:188-212.

25 Australian Bureau of Statistics. Preschool education, Australia, 2019. Available: https://www.abs.gov.au/AUSSTATS/abs@.nsf/Lookup/ 4240.0Main+Features12019? OpenDocument

26 Australian Government Department of Education Employment and Workplace Relations. Belonging, Being \& Becoming: The Early Years Framework for Australia, 2009. Available: http://www.deewr.gov.au/ Earlychildhood/Policy_Agenda/Quality/Documents/Final\%20EYLF\% 20Framework\%20Report\%20-\%20WEB.pdf

27 Childcare \& Kindergarten Association (C\&K). Become a C\&K Branch Service: C\&K Childcare and Kindergarten, 2019. Available: https:// www.candk.asn.au/become-ck-branch-service

28 Childcare \& Kindergarten Association (C\&K). Become a C\&K Affiliate Service: C\&K Childcare and Kindergarten, 2019. Available: https:// www.candk.asn.au/become-ck-affiliate-service

29 Australian Bureau of Statistics. Socio-economic indexes for areas. Canberra, Australia: Australian Bureau of Statistics, 2018. https:// www.abs.gov.au/websitedbs/censushome.nsf/home/seifa

30 Australian Government Department of Education and Training. Australian early development census data explorer: Australian government department of education and training, 2019. Available: https://www.aedc.gov.au/data/data-explorer

31 Hemmeter ML, Snyder PA, Fox L, et al. Evaluating the Implementation of the Pyramid Model for Promoting SocialEmotional Competence in Early Childhood Classrooms. Topics Early Child Spec Educ 2016;36:133-46.

32 Kline R. Principles and practice of structural equation modelling. 4th edn. New York, USA: Guildford Press, 2014.

33 Feingold $A$. Effect sizes for growth-modeling analysis for controlled clinical trials in the same metric as for classical analysis. Psychol Methods 2009;14:43-53.

34 Mun EY, von Eye A, White HR. An SEM approach for the evaluation of intervention effects using pre-post-post designs. Struct Equ Modeling 2009;16:315-37.

35 Williams KE, Barrett MS, Welch GF, et al. Associations between early shared music activities in the home and later child outcomes: findings from the longitudinal study of Australian children. Early Child Res Q 2015;31:113-24.

36 Quach JL, Nguyen CD, Williams KE, et al. Bidirectional associations between child sleep problems and internalizing and Externalizing difficulties from preschool to early adolescence. JAMA Pediatr 2018;172:e174363

37 The LSAC Research Consortium. The longitudinal study of Australian children data dictionary: Australian Institute of family studies, 2019. Available: https://growingupinaustralia.gov.au/data-anddocumentation/data-dictionary/downloads

38 Barrett MS, Flynn LM, Brown JE, et al. Beliefs and values about music in early childhood education and care: perspectives from practitioners. Front Psychol 2019;10:724.

39 Howard SJ, Melhuish E. An early years toolbox for assessing early executive function, language, self-regulation, and social development: validity, reliability, and preliminary norms. $J$ Psychoeduc Assess 2017;35:255-75.

40 Ponitz CC, McClelland MM, Matthews JS, et al. A structured observation of behavioral self-regulation and its contribution to kindergarten outcomes. Dev Psychol 2009;45:605-19.

41 Bronson MB, Tivnan T, Seppanen PS. Relations between teacher and classroom activity variables and the classroom behaviors of prekindergarten children in chapter 1 funded programs. J Appl Dev Psychol 1995;16:253-82.

42 Bracken B. Bracken school readiness assessment. 3rd edn, 2007

43 Harrison PL. Early screening profiles: manual. Circle Pines, MN: American Guidance Service, 1990.

44 Hawes DJ, Dadds MR. Australian data and psychometric properties of the strengths and difficulties questionnaire. Aust N Z J Psychiatry 2004;38:644-51. 